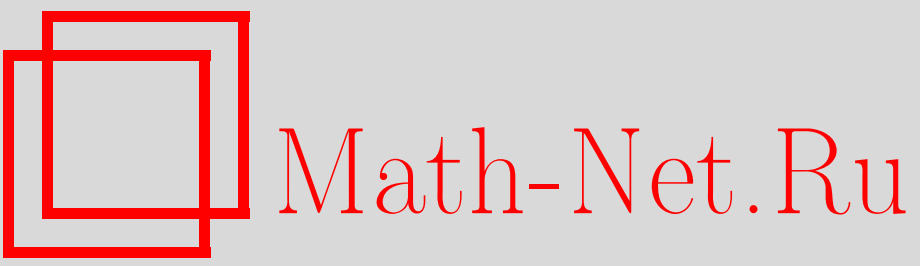

Е. А. Жижина, Асимптотическая формула для убывания корреляций в стохастической модели плоских ротаторов при высоких температурах, ТМФ, 1997, том 112, номер 1, 67-80

DOI: https://doi.org/10.4213/tmf1027

Использование Общероссийского математического портала Math-Net.Ru подразумевает, что вы прочитали и согласны с пользовательским соглашением

http: //www . mathnet.ru/rus/agreement

Параметры загрузки:

IP : 54.210 .77 .194

26 апреля 2023 г., 13:33:22

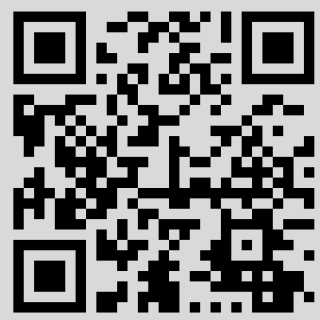




\section{Е. А. Жижина* \\ АСИМПТОТИЧЕСКАЯ ФОРМУЛА ДЛЯ УБЫВАНИЯ КОРРЕЛЯЦИЙ В СТОХАСТИЧЕСКОЙ МОДЕЛИ ПЛОСКИХ РОТАТОРОВ ПРИ ВЫСОКИХ ТЕМПЕРАТУРАХ}

\footnotetext{
Найдена асимптотическая формула для убывания корреляций $\left\langle f_{A}(x(0)), g_{A+k(t)}(x(t))\right\rangle$, когда $t \rightarrow \infty, \quad k(t) \rightarrow \infty, k(t) \in Z^{d}$, в стохастической модели плоских ротаторов $x(t)=\left\{x_{k}(t), k \in Z^{d}\right\}, t \geq 0, \quad x_{k}(t) \in T^{1}$ при высоких температурах. Основными методами исследования являются спектральный анализ генератора соответствующей марковской полугруппы, а также метод перевала.
}

\section{1. ОПИСАНИЕ МОДЕЛИ И ОСНОВНОЙ РЕЗУЛЬТАТ}

В этой работе рассматривается стохастическая (глауберова) динамика системы плоских ротаторов на решетке $Z^{d}$ при высоких температурах $T$. Основным методом исследования данной модели является спектральный анализ генератора $H_{\beta} \quad\left(\beta=T^{-1}\right.$ обратная температура) соответствуюшей марковской полугруппы $\mathcal{T}_{t}, t \geq 0$. Такой подход к изучению стохастической динамики бесконечночастичных систем был впервые развит в работах Минлоса [1], Кондратьева и Минлоса [2]. Этот метод позволяет выделить несколько первых инвариантных подпространств и изучить спектр генератора $H_{\beta}$ на этих подпространствах. Причем построение инвариантных подпространств стохастической динамики базируется на схеме выделения инвариантных подпространств кластерных операторов, изложенной в книге Малышева и Минлоса [3]. В данной работе будут использованы эти построения, а также методы вычисления асимптотического убывания корреляций для спиновых полей, развитые в работе Минлоса и Жижиной [4].

Как известно, при малых $\beta, 0<\beta \leq \beta_{0}(d)$, на пространстве всех конфигураций $\Omega=T^{Z^{d}} \ni x=\left\{x_{k}, k \in Z^{d}\right\}, x_{k} \in T$ ( $T$ - одномерный тор), сушествует единственное предельное гиббсовское распределение $\mu_{\beta}$, которое можно определить как слабый предел конечных распределений $\mu_{\Lambda, \beta}, \Lambda \subset Z^{d}$ на $\Omega$ :

$$
\mu_{\beta}=\lim _{\Lambda \nearrow Z^{d}} \mu_{\Lambda, \beta},
$$

заданных формулой

$$
d \mu_{\Lambda, \beta}=\frac{1}{Z_{\Lambda, \beta}} e^{-\beta U_{\Lambda}\left(x_{\Lambda}\right)} d \mu_{\Lambda, 0}
$$

${ }^{*}$ Институт проблем передачи информации РАН, Москва, Россия. E-mail: ejj@ippi.ac.msk.su 
Здесь $\Lambda \subset Z^{d}$ - конечные подмножества решетки, $Z_{\Lambda, \beta}$ - нормируюший множитель, $U_{\Lambda}\left(x_{\Lambda}\right)$ - гамильтониан модели плоских ротаторов в объеме $\Lambda \subset Z^{d}$ :

$$
U_{\Lambda}(x)=\sum_{k, m \in \Lambda:|k-m|=1} \cos \left(x_{k}-x_{m}\right),
$$

$d \mu_{\Lambda, 0}=\prod_{k \in \Lambda} d \mu_{0}^{(k)}=\left(d \mu_{0}\right)^{|\Lambda|}, d \mu_{0}-$ нормированная мера Хаара на торе $T$.

Введем дифференциальный оператор $H_{\beta}$ на пространстве финитных функций $D^{0}\left(H_{\beta}\right) \subset L_{2}\left(\Omega, \mu_{\beta}\right)$ по формуле

$$
\left(H_{\beta} f\right)(x)=-\sum_{k \in Z^{d}} \frac{\partial^{2} f(x)}{\partial x_{k}^{2}}-\sum_{k \in Z^{d}} b_{k}(x) \frac{\partial f(x)}{\partial x_{k}},
$$

где

$$
b_{k}(x)=-\beta \sum_{m:|k-m|=1} \sin \left(x_{k}-x_{m}\right), \quad f(x) \in D^{0}\left(H_{\beta}\right) .
$$

Как показано в работе [5], $H_{\beta}$ является сушественно самосопряженным оператором в $L_{2}\left(\Omega, \mu_{\beta}\right)$, и, следовательно, его замыкание $\bar{H}_{\beta}$ - самосопряженный оператор. В дальнейшем нам будет удобно сохранить обозначение $H_{\beta}$ для замыкания оператора (2).

В работе [6] построен обратимый марковский процесс на $\Omega$ :

$$
x(t)=\left\{x_{k}(t), k \in Z^{d}\right\}, \quad t \geq 0, \quad x_{k}(t) \in T,
$$

со стационарным распределением $\mu_{\beta}$, который порождает стохастическую полугруппу $\mathcal{T}_{t}=e^{-t H_{\beta}}, t \geq 0$, действующую в пространстве $L_{2}\left(\Omega, \mu_{\beta}\right)$ по формуле

$$
\left(\mathcal{T}_{t} f\right)(x)=\langle f(x(t))\rangle_{\mathcal{P}, x}, \quad x \in \Omega
$$

где \langle\rangle$_{\mathcal{P}, x}$ - условное среднее относительно распределения вероятностей $\mathcal{P}$ процесса $x(t)$ при условии, что $x \in \Omega$ - начальная конфигурация: $x(0)=x$.

Далее рассмотрим гладкие функции, зависящие от конечного числа переменных,

$$
f_{A}(x) \equiv f_{A}\left(x_{a_{1}}, \ldots, x_{a_{m}}\right), \quad x \in \Omega, \quad x_{a_{j}} \in T
$$

(здесь $A=\left\{a_{1}, \ldots, a_{m}\right\} \subset Z^{d}$ - конечное подмножество решетки $Z^{d}$ ), которые обладают непрерывными производными порядка $s_{A}=[m / 2]+1$, где $m=|A|,[m / 2]$ - целая часть числа $m / 2: f_{A}(x) \in C^{s_{A}}\left(T^{|A|}\right)$. В этой работе при дополнительном условии на такие функции, которое будет сформулировано ниже, получена асимптотическая формула для убывания коррелящий вида

$$
\begin{aligned}
I_{f, g}(t, k(t)) & \equiv\left\langle f_{A}(x(0)), g_{A+k(t)}(x(t))\right\rangle_{\mathcal{P}}= \\
& =\left\langle f_{A}(x(0)) g_{A+k(t)}(x(t))\right\rangle_{\mathcal{P}}-\left\langle f_{A}(x(0))\right\rangle_{\mathcal{P}}\left\langle g_{A+k(t)}(x(t))\right\rangle_{\mathcal{P}},
\end{aligned}
$$

когда $t \rightarrow \infty, k(t) \rightarrow \infty, k(t) \in Z^{d}$. Здесь \langle\rangle$_{\mathcal{P}}-$ среднее относительно распределения вероятностей $\mathcal{P}$ процесса $x(t)$.

Нам понадобится наложить ряд условий на рост вектора $k(t)$ при $t \rightarrow \infty$. Фиксируем некоторое $0<\beta \leq \beta_{0}(d)$. Мы предположим, что

$$
\frac{k_{j}(t)}{t} \rightarrow u_{j} \quad \text { при } \quad t \rightarrow \infty, \quad j=1, \ldots, d,
$$


причем

$$
\frac{k_{j}(t)}{t}-u_{j}=\alpha_{j}(t)=o\left(\frac{1}{\sqrt{t}}\right), \quad t \rightarrow \infty,
$$

и

$$
|u|=\sum_{j=1}^{d}\left|u_{j}\right|<\frac{1}{|\ln \beta|}, \quad u=\left(u_{1}, \ldots, u_{d}\right),
$$

при каждом фиксированном $0<\beta \leq \beta_{0}(d)$.

Теперь мы можем сформулировать основной результат.

ТЕОрема 1. Пусть $r(t)=(t, k(t)) \in R \times Z^{d}$ и пусть вектор $k(t)$ удовлетворяет условиям (3)-(5). Тогда существует такой вектор $p=p(u, \beta) \in R^{d+1}$, что имеет место следующая асимптотическая формула:

$$
I_{f, g}(t, k(t))=\frac{K_{f, g}^{d}(u)}{|r(t)|^{d / 2}} e^{-(r(t), p)}(1+o(1)), \quad t \rightarrow \infty .
$$

Здесь $K_{f, g}^{d}(u)$ - константа, зависящая от функций $f(x), g(x)$ и вектора $u \in Z^{d}$, $(r(t), p)$ - скалярное произведение.

ЗАмЕчАниЕ 1 . В случае, если координаты вектора $u$ удовлетворяют условию

$$
\beta^{1-\varepsilon}<\sum_{j=1}^{d}\left|u_{j}\right|<\frac{1}{|\ln \beta|}
$$

где $0<\varepsilon<1$ - любая сколь угодно малая константа, вектор $p=p(u, \beta) \in R^{d+1}$ допускает следующее представление:

$$
p=p_{0}(u, \beta)+p_{1}(u, \beta),
$$

здесь

$$
\begin{aligned}
& p_{0}=\left(1-\beta \sum_{j=1}^{d} \cosh \mu_{j}, \mu\right), \quad \mu \in R^{d}, \quad p_{0} \in R^{d+1}, \\
& \mu_{j}=\operatorname{sign}\left(u_{j}\right) \ln \frac{2\left|u_{j}\right|}{\beta}, \quad j=1, \ldots, d, \quad\left|\left(p_{1}(u, \beta)\right)_{j}\right| \leq C(\beta / u)^{2}, \quad j=1, \ldots, d+1, \\
& C \text { - некоторая абсолютная константа. }
\end{aligned}
$$

\section{2. ОСНОВНЫЕ КОНСТРУКЦИИ И ФАКТЫ}

В этом разделе мы опишем одночастичные инвариантные подпространства $\mathcal{H}_{+}$и $\mathcal{H}_{-}$ оператора $H_{\beta}$, построенные в работе [2], и найдем спектр $H_{\beta}$ на этих подпространствах. При этом для доказательства теоремы 1 нам понадобятся более точные, чем в [2], оценки спектра оператора $H_{\beta}$, ограниченного на подпространствах $\mathcal{H}_{ \pm}$.

Обозначим через $\mathcal{H}=L_{2}\left(\Omega, \mu_{\beta}\right), \Omega=\left\{x_{k} \in T, k \in Z^{d}\right\}$, пространство квадратично интегрируемых функций на множестве $\Omega$ по мере $\mu_{\beta}$, а через $\mathcal{M}$ - множество всех целочисленных функций (мультииндексов) с конечным носителем $\mathcal{M}=\{n=(n(k))$, $\left.k \in Z^{d}\right\}$, где $n(k) \in Z$ и $n(k)=0$ при $|k|>N(n)$. Тогда множество функций 


$$
L_{\mathcal{M}}=\left\{e_{n}(x)=\prod_{k \in \operatorname{supp} n} e^{i n(k) x_{k}}, n \in \mathcal{M}\right\}
$$

образует (неортогональный) базис в $\mathcal{H}$. Обозначим далее через

$$
r(n)=\sum_{k \in \operatorname{supp} n} n(k)
$$

заряд функции $n \in \mathcal{M}$. Заметим, что построенная выше стохастическая динамика сохраняет заряд, следовательно, все пространство $\mathcal{H}$ распадается в прямую сумму инвариантных подпространств

$$
\mathcal{H}=\oplus \sum_{r \in Z} \mathcal{H}_{r}
$$

причем каждое $\mathcal{H}_{r}$ образовано векторами вида $e_{n}(x)$, где $r(n)=r$. Таким образом, подпространства $\mathcal{H}_{ \pm}$, которые строятся как возмушение соответствуюших подпространств

$$
\mathcal{H}_{ \pm}^{0}=\left\{\sum_{k \in Z^{d}} c_{k} e^{ \pm i x_{k}}\right\}
$$

лежат в подпространствах $\mathcal{H}_{ \pm 1}$ соответственно: $\mathcal{H}_{+} \subset \mathcal{H}_{1}, \mathcal{H}_{-} \subset \mathcal{H}_{-1}$. Обозначим через $U_{j}, j \in Z^{d}$, унитарную группу сдвигов, действуюшую в пространстве $\mathcal{H}$ по формуле

$$
\left(U_{j} f\right)(x)=f\left(x^{(j)}\right),
$$

где $\left(x^{(j)}\right)_{k}=x_{k-j}-$ сдвиг конфигуурации $x \in \Omega$ на вектор $j \in Z^{d}$. Пусть $(J f)(x)=$ $f(-x)$ - унитарная инволюция в $\mathcal{H}$. В работе [2] доказано, что при малых $\beta$ существуют два взаимно ортогональных подпространства $\mathcal{H}_{ \pm} \subset \mathcal{H}$, инвариантных относительно операторов $H_{\beta}$ и $U_{j}, j \in Z^{d}$. При этом оператор $J$ устанавливает взаимно однозначное соответствие между этими подпространствами: $J \mathcal{H}_{ \pm}=\mathcal{H}_{\mp}$.

Спектры операторов $H_{+}=\left.H_{\beta}\right|_{\mathcal{H}_{+}}$и $H_{-}=\left.H_{\beta}\right|_{\mathcal{H}_{-}}$совпадают. Эти операторы имеют абсолютно непрерывньй спектр, представляюший собой множество значений функции

$$
m(\lambda)=1-\beta \sum_{j=1}^{d} \cos \lambda_{j}+m_{1}(\lambda),
$$

где $\left|m_{1}(\lambda)\right| \leq C_{1} \beta^{2}, \lambda \in W$ (комплексная окрестность тора $T^{d}$ ):

$$
W=\left\{\left|\operatorname{Im} \lambda_{j}\right|<\log 3 / 2, \quad j=1, \ldots, d\right\} .
$$

Для спектра оператора $H_{\beta}$ в ортогональном дополнении

$$
\mathcal{H}^{\perp}=\mathcal{H} \ominus\left\{\mathcal{H}_{0} \oplus \mathcal{H}_{+} \oplus \mathcal{H}_{-}\right\},
$$

где $\mathcal{H}_{0}$ - пространство констант, справедливо неравенство

$$
\sigma\left(\left.H_{\beta}\right|_{\mathcal{H}^{\perp}}\right)>2-C_{2} \beta
$$

где $C_{2}$ - абсолютная константа. 
Обозначим через

$$
W_{\beta}=\left\{\left|\operatorname{Im} \lambda_{j}\right|<-\ln \beta-\ln D, \quad j=1, \ldots, d\right\}
$$

комплексную окрестность тора $T^{d}$, где $D=D(d)$ - некоторая константа, зависящая только от размерности $d: D>\frac{13}{2} d$. Такой выбор этой константы будет пояснен ниже. Оказывается, что функция $m(\lambda)$ сохраняет свое представление в области $W_{\beta}$, а именно, справедлива теорема 2.

ТЕОрема 2. Функиия $m(\lambda)$ имеет следующее представление:

$$
m(\lambda)=1-\beta \sum_{j=1}^{d} \cos \lambda_{j}+m_{1}(\lambda)=m_{0}(\lambda)+m_{1}(\lambda),
$$

где функиия $m_{1}(\lambda)$ аналитически продолжсается внутрь полось $W_{\beta}$ и является равномерно ограниченной вместе со всеми своими производными:

$$
\left|m_{1}(\lambda)\right| \leq B \beta^{2}, \quad\left|D^{\alpha} m_{1}(\lambda)\right| \leq B_{\alpha} \beta^{2},
$$

внутри полосы

$$
\left\{\left|\operatorname{Im} \lambda_{j}\right|<-\ln \beta-\ln \frac{D}{\gamma}, \quad j=1, \ldots, d\right\}
$$

для любого $0<\gamma<1$. Здесь $B=B(\gamma, D, d) ; \quad B_{\alpha}=B_{\alpha}(\gamma, D, d)-$ константы, зависящие от $\gamma, D, d ; \alpha=\left(\alpha_{1}, \ldots, \alpha_{d}\right)$ - мультииндекс,

$$
D^{\alpha}=\frac{\partial^{\alpha_{1}+\cdots+\alpha_{d}}}{\partial \lambda_{1}^{\alpha_{1}} \ldots \partial \lambda_{d}^{\alpha_{d}}} .
$$

Доказательство этой теоремы основывается на общей схеме построения первых инвариантных подпространств для операторов в бесконечночастичных системах [3]. Поэтому вначале мы введем все необходимые конструкции, адаптированные к данной модели, и сформулируем леммы, на которых будет базироваться доказательство.

Для каждой функции $n \in \mathcal{M}$ определим величину

$$
|n|=\sum_{k \in \operatorname{supp} n}|n(k)|,
$$

и пусть

$$
\mathcal{M}_{1}^{ \pm}=\{n \in \mathcal{M}:|n|=1, r(n)= \pm 1\}, \quad \mathcal{M}_{>1}^{ \pm}=\{n \in \mathcal{M}:|n|>1, r(n)= \pm 1\} .
$$

Обозначим через $L_{1}^{ \pm}$линейную оболочку, натянутую на векторы $\left\{e_{n}, n \in \mathcal{M}_{1}^{ \pm}\right\}$, а через $L_{>1}^{ \pm}$- линейную оболочку векторов $\left\{e_{n}, n \in \mathcal{M}_{>1}^{ \pm}\right\}$, тогда

$$
\mathcal{H}_{ \pm 1}=L_{1}^{ \pm}+L_{>1}^{ \pm}
$$

и будем строить инвариантные подпространства $\mathcal{H}_{ \pm}$в виде

$$
\mathcal{H}_{ \pm}=L_{1}^{ \pm}+S^{ \pm} L_{1}^{ \pm},
$$


где $S^{ \pm}: L_{1}^{ \pm} \rightarrow L_{>1}^{ \pm}$- операторы, существование и единственность которых в некотором классе операторов будет доказана ниже.

Далее мы проведем все доказательство для подпространства $\mathcal{H}_{+}$(случай $\mathcal{H}_{-}$рассматривается аналогично). Разложение (12) позволяет переписать оператор $\left.H_{\beta}\right|_{\mathcal{H}_{1}}$ в виде матрищы

$$
\left(\begin{array}{ll}
H_{00} & H_{01} \\
H_{10} & H_{11}
\end{array}\right)
$$

где $H_{00}: L_{1}^{+} \rightarrow L_{1}^{+}, H_{01}: L_{>1}^{+} \rightarrow L_{1}^{+}$и т.д. В частности, как легко следует из формулы $(2)$ для оператора $H_{\beta}$, имеем

$$
\left(H_{00}\right)_{k, n}= \begin{cases}1, & k=n \\ -\beta / 2, & |k-n|=1 \\ 0, & |k-n| \geq 2 .\end{cases}
$$

Требование инвариантности подпространства $\mathcal{H}_{+}$относительно оператора $H_{\beta}$ переписывается в виде следуюшего уравнения на оператор $S=S^{+}$:

$$
S=H_{11}^{-1} S H_{00}+H_{11}^{-1} S H_{01} S-H_{11}^{-1} H_{10} .
$$

Рассмотрим подпространство $A_{D}$ ограниченных операторов $Q: L_{1}^{+} \rightarrow L_{1}^{+}$, удовлетворяюших условию

$$
\|Q\|_{D}=\sup _{k} \sum_{n}\left|Q_{k, n}\right| \frac{1}{(D \beta)^{|k-n|}}<\infty,
$$

где $D$ - некоторая константа. Легко проверить, что справедливо следующее неравенство:

$$
\left\|Q_{1} Q_{2}\right\|_{D} \leq\left\|Q_{1}\right\|_{D}\left\|Q_{2}\right\|_{D}
$$

Действительно,

$$
\begin{aligned}
\left\|Q_{1} Q_{2} \mid\right\|_{D} & =\sup _{k} \sum_{n}\left|\left(Q_{1} Q_{2}\right)_{k, n}\right| \frac{1}{(D \beta)^{|k-n|}} \leq \\
& \leq \sup _{k} \sum_{n, m}\left|\left(Q_{1}\right)_{k, m}\right|\left|\left(Q_{2}\right)_{m, n}\right| \frac{1}{(D \beta)^{|k-m|}(D \beta)^{|m-n|}} \leq \\
& \leq \sup _{k} \sum_{m} \frac{\left|\left(Q_{1}\right)_{k, m}\right|}{(D \beta)^{|k-m|}} \sup _{m} \sum_{n} \frac{\left|\left(Q_{2}\right)_{m, n}\right|}{(D \beta)^{|m-n|}}=||\left|Q_{1}\right|\left\|_{D}|| Q_{2} \mid\right\|_{D}
\end{aligned}
$$

ЛЕмма 1. При $D>\frac{13}{2} d$ существует решение $S$ уравнения (16), при этом опеpamop $H_{01} S \in A_{D} u$

$$
\left\|H_{01} S\right\|_{D} \leq C \beta^{2} .
$$

Другими словами, матричные әлементы оператора $H_{01} S$ допускают следующую оценку:

$$
\left|\left(H_{01} S\right)_{k, n}\right| \leq \beta^{2} d_{k, n}(D \beta)^{|k-n|},
$$

$2 \partial e$

$$
\sup _{k} \sum_{n}\left|d_{k, n}\right|<C, \quad C=C(D, d)=\frac{3 D d}{2 D-13 d}
$$


ДокАЗАтЕльство. Введем в фактор-пространстве ограниченных операторов $\mathcal{L}=$ $\left\{W: L_{1}^{+} \rightarrow L_{>1}^{+}\right\}$норму $\|\left.|\cdot|\right|_{H}$, индуцированную нормой $\left|\|\cdot \mid\|_{D}\right.$, по формуле

$$
\||W|\|_{H} \equiv\left\|H_{01} W\right\|_{D},
$$

и пусть $\mathcal{L}_{H}=\left\{W \in \mathcal{L}:|||W| \|_{H}<\infty\right\}$. Перепишем уравнение (16) в виде

$$
S=F S=-H_{11}^{-1} H_{10}+H_{11}^{-1} S H_{00}+H_{11}^{-1} S H_{01} S,
$$

и пусть

$$
\mathcal{B}=\left\{W \in \mathcal{L}_{H}:\|W \mid\|_{H}<C(d, D) \cdot \beta^{2}\right\}
$$

- некоторый шар в $\mathcal{L}_{H}$. Здесь $C(d, D)=\frac{3 D d}{2 D-13 d}$. Мы покажем, что отображение $F$ при соответствуюшем выборе константы $D=D(d)$ переводит шар $\mathcal{B}$ в себя: $F \mathcal{B} \subset \mathcal{B}$, и является сжимаюшим внутри $\mathcal{B}$. Отсюда будет следовать, что уравнение (19) имеет единственное решение $S$, лежашее внутри $\mathcal{B}$.

Для этого нам нужно оценить нормы всех операторов, входящих в выражение (19). Пользуясь формулой (2) для оператора $H_{\beta}$, запишем разложение для оператора $H_{11}^{-1}$ в виде ряда

$$
H_{11}^{-1}=\left(h\left(1-h^{-1} \gamma\right)\right)^{-1}=h^{-1} \sum_{p=0}^{\infty} V^{p}
$$

где $h=\operatorname{diag}\left\{h_{n}\right\}_{n \in \mathcal{M}_{>1}^{+}}-$диагональная матрица со значениями $h_{n} \geq 3, n \in \mathcal{M}_{>1}^{+}$, $h_{n} \in \mathbf{N}, V=h^{-1} \gamma$, причем все матричные элементы оператора $V$ равномерно ограничены:

$$
\left|V_{n, n^{\prime}}\right| \leq \beta, \quad n, n^{\prime} \in \mathcal{M}_{>1}^{+} .
$$

Имеет место следуюшая оценка, доказательство которой проводится аналогично тому, как это сделано в работе [2].

Пусть $R: L_{1}^{+} \rightarrow L_{>1}^{+}, R \in \mathcal{L}_{H}$. Тогда справедлива оценка

$$
\left|\left(H_{01} V^{p} R\right)_{k, n}\right| \leq\left(\frac{4 d}{D}\right)^{p}(D \beta)^{|k-n|} d_{k, n},
$$

где

$$
\sup _{k} \sum_{n}\left|d_{k, n}\right| \leq|| \mid H_{01} R \|_{D}
$$

Из оценки (20) и формулы (15) для оператора $H_{00}$ сразу следует неравенство

$$
\begin{aligned}
& \left|\left(H_{01} H_{11}^{-1} S H_{00}\right)_{k, n}\right| \leq \frac{1}{3} \sum_{p=0}^{\infty}\left|\left(H_{01} V^{p} S H_{00}\right)_{k, n}\right| \leq \\
& \quad \leq \frac{1}{3} \sum_{p=0}^{\infty}\left|\left(H_{01} V^{p} S\right)_{k, n}\right|+\frac{\beta}{6} \sum_{p=0}^{\infty} \sum_{j=1}^{d}\left|\left(H_{01} V^{p} S\right)_{k \pm e_{j}, n}\right|< \\
& \quad<\frac{1}{3}(D \beta)^{|k-n|} \sum_{p=0}^{\infty}\left(\frac{4 d}{D}\right)^{p} d_{k, n}+\frac{\beta d}{3}(D \beta)^{|k-n|-1} \sum_{p=0}^{\infty}\left(\frac{4 d}{D}\right)^{p} d_{k, n}= \\
& \quad=\frac{1}{3}\left(1+\frac{d}{D}\right)(D \beta)^{|k-n|} d_{k, n} \sum_{p=0}^{\infty}\left(\frac{4 d}{D}\right)^{p},
\end{aligned}
$$


следовательно,

$$
\left\|\left|\left(H_{01} H_{11}^{-1} S H_{00}\right)\left\|_{D}<\alpha_{1}(d, D) \mid\right\| H_{01} S \|_{D},\right.\right.
$$

где

$$
\alpha_{1}(d, D)=\frac{1}{3}\left(1+\frac{d}{D}\right) \sum_{p=0}^{\infty}\left(\frac{4 d}{D}\right)^{p},
$$

и при $D>\frac{13}{2} d$ имеем $0<\alpha_{1}(d, D)<1$.

Далее, пользуясь неравенствами (17) и (20), получаем следующую оценку:

$$
\left\|H _ { 0 1 } H _ { 1 1 } ^ { - 1 } S H _ { 0 1 } S | \| _ { D } \leq \| | H _ { 0 1 } H _ { 1 1 } ^ { - 1 } S | \| _ { D } \| | H _ { 0 1 } S \left|\left\|_{D} \leq \alpha_{2}(d, D)\right\|\left\|H_{01} S \mid\right\|_{D}^{2},\right.\right.
$$

где

$$
\alpha_{2}(d, D)=\frac{1}{3} \sum_{p=0}^{\infty}\left(\frac{4 d}{D}\right)^{p}<1
$$

при $D>6 d$. Аналогично находим

$$
\left\|\left|H_{01} H_{11}^{-1} H_{10}\right|\right\|_{D} \leq \alpha_{2}(d, D)\|\| H_{01} H_{10} \|_{D} \leq \alpha_{2}(d, D) \frac{3}{2} d \beta^{2} .
$$

Таким образом, окончательно имеем

$$
\|\left.|F S|\right|_{H}<\left.\alpha_{1}|||S|\right|_{H}+\left.\alpha_{2}|||S|\right|_{H} ^{2}+\alpha_{2} \frac{3}{2} d \beta^{2} .
$$

Из этого неравенства, а также из выражений $(21),(22)$ для констант $\alpha_{1}$ и $\alpha_{2}$ следует, что при малых $\beta$ отображение $F$ таково, что $F \mathcal{B} \subset \mathcal{B}$. Кроме того, из представления (19) для отображения $F$ заключаем, что

$$
F S_{1}-F S_{2}=H_{11}^{-1}\left(S_{1}-S_{2}\right) H_{00}+H_{11}^{-1}\left(S_{1}-S_{2}\right) H_{01} S_{1}+H_{11}^{-1} S_{2} H_{01}\left(S_{1}-S_{2}\right),
$$

а из приведенных выше оценок получаем, что для $S_{1}, S_{2} \in \mathcal{B}$ справедливо неравенство

$$
\left\|F S_{1}-F S_{2}\left|\left\|_{H}<\alpha_{1}\right\|\right| S_{1}-S_{2}\right\|_{H}+2 \alpha_{2} C(d, D) \beta^{2}\left\|\mid S_{1}-S_{2}\right\|_{H} .
$$

Так как при всех достаточно малых $\beta$

$$
\alpha_{1}+2 \alpha_{2} C(d, D) \beta^{2}<1
$$

то отображение $F$ является сжимаюшим относительно нормы ||$|\cdot|||_{H}$ на шаре $\mathcal{B}$ и, следовательно,

$$
\||S|\|_{H}=\left|\left\|H_{01} S \mid\right\|_{D} \leq C(d, D) \beta^{2} .\right.
$$

Лемма 1 доказана.

Таким образом, мы доказали сушествование инвариантного подпространства $\mathcal{H}_{+} \subset \mathcal{H}_{1}$, которое имеет вид $(13)$, где оператор $S^{+}$удовлетворяет условиям леммы 1. Аналогичным образом доказывается сушествование инвариантного подпростарнства $\mathcal{H}_{-}=L_{1}^{-}+S^{-} L_{1}^{-}$.

Следовательно, в пространстве $\mathcal{H}_{+}$сушествует базис, состояший из векторов вида

$$
v_{k}=e_{k}+S^{+} e_{k}, \quad k \in Z^{d}
$$


где $e_{k}=e^{i x_{k}}, x_{k} \in T$. Так как оператор $H_{+}$коммутирует с группой пространственных сдвигов $U_{j}, j \in Z^{d}$, то оператор $H_{+}$является оператором свертки и действует по формуле

$$
H_{+} v_{k}=\sum_{n} m(k-n) v_{n}
$$

где

$$
m(k-n)=\left(H_{00}\right)_{k-n}+\left(H_{01} S\right)_{k-n},
$$

а операторы $H_{00}, H_{01}$ введены соотношениями (14), (15). Кроме того, из леммы 1 следует, что

$$
\left|\left(H_{01} S\right)_{r}\right| \leq \beta^{2}(D \beta)^{|r|} d_{r}, \quad r=k-n \in Z^{d},
$$

где $\sum_{r}\left|d_{r}\right|<C=C(d, D)$. Таким образом, можно записать функцию $m(r)$ в виде

$$
m(r)=m_{0}(r)+m_{1}(r), \quad r \in Z^{d},
$$

где функция $m_{0}(r)=\left(H_{00}\right)_{r}=\left(H_{00}\right)_{k, n}, r=k-n$, определена формулой $(15)$, а $m_{1}(r)=\left(H_{01} S\right)_{r}$.

Далее воспользуемся следующей леммой из работы [2].

Лемма 2. Пусть $\left\{w_{k}, k \in Z^{d}\right\}$ - ортонормированныц базис в $\mathcal{H}_{+}$, построенный из базиса (23) с помощью матрищь Грамма

$$
G=\left\{g_{k-n}\right\}_{k, n \in Z^{d}}, \quad g_{k-n}=\left(v_{k}, v_{n}\right)_{\mathcal{H}},
$$

и пусть функция $\widetilde{m}(k-n)$ определяет действие оператора $H_{+}$в этом базисе:

$$
H_{+} w_{k}=\sum_{n} \tilde{m}(k-n) w_{n}
$$

Тогда $\widetilde{m}(r)=m(r), \quad r=k-n \in Z^{d}$.

ДокАЗАтЕЛЬСтво теоремы 2. Рассмотрим преобразование Фурье $V_{+}$, переводящее пространство $\mathcal{H}_{+}$в $L_{2}\left(T^{d}, d \lambda_{0}\right)$ :

$$
V_{+}: w_{k} \rightarrow e^{i(k, \lambda)}, \quad k \in Z^{d}, \quad \lambda \in T^{d},
$$

где $T^{d}-d$-мерный тор, $d \lambda_{0}$ - нормированная мера, при этом

$$
\left(V_{+} U_{j}\right) f(\lambda)=e^{i(j, \lambda)} f(\lambda), \quad j \in Z^{d}, \quad f(\lambda) \in L_{2}\left(T^{d}, d \lambda_{0}\right),
$$

а оператор $H_{+}$переходит в оператор $\widetilde{H}_{+}$умножения на функцию $m(\lambda)$ :

$$
\left(\widetilde{H}_{+} f\right)(\lambda)=m(\lambda) f(\lambda) .
$$

В силу леммы 2

$$
m(\lambda)=\sum_{r \in Z^{d}} m(r) e^{i(r, \lambda)},
$$

а функция $m(r)$ определена равенством (24). Из (15) и (24) следует, что

$$
m(\lambda)=1-\beta \sum_{j=1}^{d} \cos \lambda_{j}+m_{1}(\lambda),
$$


где $m_{1}(\lambda)=\sum_{r \in Z^{d}}\left(H_{01} S\right)_{r} e^{i(r, \lambda)}$. Воспользовавшись далее леммой 1, имеем, что при

$$
\left|\operatorname{Im} \lambda_{j}\right|<-\ln \beta-\ln \frac{D}{\gamma}, \quad 0<\gamma<1, \quad j=1, \ldots, d,
$$

выполняется следуюшая цепочка неравенств:

$$
\begin{aligned}
\left|m_{1}(\lambda)\right| & \leq \sum_{r \in Z^{d}}\left|\left(H_{01} S\right)_{r}\right|\left(\frac{\gamma}{D \beta}\right)^{|r|} \leq \beta^{2} \sum_{|r|=0}^{\infty}\left(\frac{\gamma}{D \beta}\right)^{|r|}(D \beta)^{|r|} \sum_{u:|u|=|r|}\left|d_{u}\right| \leq \\
& \leq \beta^{2} C(d, D) \sum_{|r|=0}^{\infty} \gamma^{|r|}=C(d, D) \beta^{2} \frac{1}{1-\gamma} .
\end{aligned}
$$

Здесь $|r|=\sum_{j=1}^{d}\left|r_{j}\right|$. Ограниченность производных $D^{\alpha} m_{1}(\lambda), \alpha=\left(\alpha_{1}, \ldots, \alpha_{d}\right)$, доказывается аналогично. Теорема 2 доказана.

\section{3. ДОКАЗАТЕЛЬСТВО ТЕОРЕМЫ 1. ПОСТРОЕНИЕ ПЕРЕВАЛЬНОГО КОНТУРА}

Разложим функции $f_{A}(x), g_{A}(x) \in C^{s_{A}}\left(T^{m}\right), A=\left\{a_{1}, \ldots, a_{m}\right\}, m=|A|$, в ряд Фурье:

$$
\begin{aligned}
& f_{A}(x)=\sum_{k_{1}, \ldots, k_{m} \in Z} f_{k_{1}, \ldots, k_{m}} \exp \left\{i\left(k_{1} x_{a_{1}}+\cdots+k_{m} x_{a_{m}}\right)\right\}, \\
& g_{A}(x)=\sum_{k_{1}, \ldots, k_{m} \in Z} g_{k_{1}, \ldots, k_{m}} \exp \left\{i\left(k_{1} x_{a_{1}}+\cdots+k_{m} x_{a_{m}}\right)\right\} .
\end{aligned}
$$

Воспользовавшись определением заряда $r(n)$ (см. (7)), можно переписать разложения (25) и (26) следуюшим образом:

$$
f_{A}(x)=\sum_{r \in Z} f_{A}(r, x), \quad g_{A}(x)=\sum_{r \in Z} g_{A}(r, x),
$$

где $f_{A}(r, x) \in \mathcal{H}_{r}, r \in Z$,

$$
f_{A}(r, x)=\sum_{k_{1}+\cdots+k_{m}=r} f_{k_{1}, \ldots, k_{m}} \exp \left\{i\left(k_{1} x_{a_{1}}+\cdots+k_{m} x_{a_{m}}\right)\right\} .
$$

Аналогичное представление имеет место для $g_{A}(r, x) \in \mathcal{H}_{r}$.

ПРЕДПОЛОЖЕНИЕ. Функиии $f_{A}(x)$ u $g_{A}(x)$ таковы, что либо $f_{A}(1, x) \times$ $g_{A}(1, x) \neq 0$, либо $f_{A}(-1, x) \cdot g_{A}(-1, x) \neq 0$.

Так как $\mathcal{H}_{ \pm} \subset \mathcal{H}_{ \pm 1}$, то можно записать для функций $f_{A}(x)$ и $g_{A}(x)$ следующие разложения:

$$
f_{A}=a_{0}+h_{f}^{+}+h_{f}^{-}+h_{f}^{\perp}, \quad g_{A}=b_{0}+h_{g}^{+}+h_{g}^{-}+h_{g}^{\perp} .
$$

Здесь $a_{0}=\left\langle f_{A}(x)\right\rangle_{\mu_{\beta}} \in \mathcal{H}_{0}, \quad b_{0}=\left\langle g_{A}(x)\right\rangle_{\mu_{\beta}} \in \mathcal{H}_{0},\langle\cdot\rangle_{\mu_{\beta}}$ - среднее относительно меры $\mu_{\beta}, h_{f, g}^{ \pm}$- проекции функций $f_{A}( \pm 1, x), g_{A}( \pm 1, x)$ на инвариантные подпространства $\mathcal{H}_{ \pm}$, соответственно, $h_{f, g}^{ \pm} \in \mathcal{H}_{ \pm}, h_{f, g}^{\perp} \in \mathcal{H}^{\perp}=\mathcal{H} \ominus\left\{\mathcal{H}_{0} \oplus \mathcal{H}_{+} \oplus \mathcal{H}_{-}\right\}$. 
Воспользовавшись разложениями (27), имеем

$$
\begin{aligned}
I_{f, g}(t, k(t))= & \left(f_{A}(x(0)), \mathcal{T}_{t} U_{k(t)} g_{A}(x(0))\right)_{\mathcal{H}}-\left\langle f_{A}(x(0))\right\rangle_{\mu_{\beta}}\left\langle g_{A}(x(0))\right\rangle_{\mu_{\beta}}= \\
= & \left(h_{f}^{+}, e^{-t H_{+}} U_{k(t)} h_{g}^{+}\right) \mathcal{H}_{+}+\left(h_{f}^{-}, e^{-t H_{-}} U_{k(t)} h_{g}^{-}\right) \mathcal{H}_{-}+ \\
& +\left(h_{f}^{\perp}, e^{-t H^{\perp}} U_{k(t)} h_{g}^{\perp}\right)_{\mathcal{H}^{\perp}} .
\end{aligned}
$$

В силу условия (8) на спектр оператора $H^{\perp}=\left.H_{\beta}\right|_{\mathcal{H}^{\perp}}$ получаем, что последнее слагаемое в разложении (28) имеет порядок

$$
\left(h_{f}^{\perp}, e^{-t H^{\perp}} U_{k(t)} h_{g}^{\perp}\right)_{\mathcal{H}^{\perp}}=O\left(e^{-t\left(2-C_{2} \beta\right)}\right) .
$$

Переходя к преобразованию Фурье , запишем два первых слагаемых в (28) в виде интегралов:

$$
\begin{aligned}
I_{f, g}(t, k(t))= & \int_{T^{d}} \tilde{h}_{f}^{+}(\lambda) \overline{\tilde{h}_{g}^{+}(\lambda)} e^{-t m(\lambda)-i(k(t), \lambda)} d \lambda+ \\
& +\int_{T^{d}} \tilde{h}_{f}^{-}(\lambda) \overline{\tilde{h}_{g}^{-}(\lambda)} e^{-t m(\lambda)-i(k(t), \lambda)} d \lambda+O\left(e^{-t\left(2-C_{2} \beta\right)}\right) .
\end{aligned}
$$

ПРЕДЛОЖЕНИЕ 1. Функиии $\tilde{h}_{f, g}^{ \pm}(\lambda)$ допускают аналитическое продолжение в полосу $W_{\beta}(9)$.

Доказательство этого предложения основывается на оценках из леммы 1.

ЗАмЕчАниЕ 2 . Как было сказано выше, унитарная инволюция $J$ устанавливает взаимно однозначное соответствие между подпространствами $\mathcal{H}_{+}$и $\mathcal{H}_{-}$, и, в частности, $h_{f, g}^{-}=J h_{f, g}^{+}$. Следовательно, $\tilde{h}_{f, g}^{-}(\lambda)=\overline{\tilde{h}_{f, g}^{+}(\lambda)}$.

Лемма 3. Пусть $k(t) \in Z^{d}$ - вектор, удовлетворяющий условиям (3)-(5), и nусть $r(t)=(t, k(t)) \in R \times Z^{d}$. Тогда существует такой вектор $p=p(u) \in R^{d+1}$, что справедлива следующая асимптотическая формула $($ при $t \rightarrow \infty)$ :

$$
\int_{T^{d}}\left(\tilde{h}_{f}^{+}(\lambda) \overline{\tilde{h}_{g}^{+}(\lambda)}\right) e^{-t m(\lambda)-i(k(t), \lambda)} d \lambda=\frac{C_{d}}{|r(t)|^{d / 2}} e^{-(r(t), p)}(1+o(1)) .
$$

Здесь $C_{d}$ - некоторая константа, которая зависит от $d, u$, а также функиий $\tilde{h}_{f, g}^{+}(\lambda)$ u $m(\lambda)$. В случае, если

$$
\beta^{1-\varepsilon}<\sum_{j=1}^{d}\left|u_{j}\right|<\frac{1}{|\ln \beta|},
$$

где $0<\varepsilon<1$ - любая константа, вектор $p \in R^{d+1}$ допускает следующее представление:

$$
p=p_{0}(u, \beta)+p_{1}(u, \beta),
$$

əдe

$$
\begin{gathered}
p_{0} \in R^{d+1}, \quad p_{0}(u, \beta)=\left(m_{0}(-i \mu), \mu\right), \quad \mu \in R^{d}, \\
\mu_{j}=\operatorname{sign}\left(u_{j}\right) \ln \frac{2\left|u_{j}\right|}{\beta}, \quad j=1, \ldots, d,
\end{gathered}
$$

а координаты вектора $p_{1}(u, \beta) \in R^{d+1}$ удовлетворяют следующей оченке с некоторой абсолютной константой $C$ :

$$
\left|\left(p_{1}(u, \beta)\right)_{j}\right|<C(\beta / u)^{2}, \quad j=1, \ldots, d+1 .
$$


ДокАЗАТЕльСтво. Так как функция

$$
\tilde{h}_{1}^{+}(\lambda)=\tilde{h}_{f}^{+}(\lambda) \overline{\tilde{h}_{g}^{+}(\lambda)}
$$

допускает аналитическое продолжение в комплексную область $W_{\beta}$, для нахождения асимптотической формулы (31) следует воспользоваться методом перевала [7]. При этом в дальнейшем мы будем полагать, что $\lambda$-комплексная переменная: $\lambda \in C^{d}$. Введем обозначение

$$
S_{t}(\lambda, k(t))=-t m(\lambda)-i(k(t), \lambda) .
$$

С помощью представления (10) для функции $m(\lambda)$ и условий (3)-(5) на вектор $k(t)$ можно записать функцию $S_{t}(\lambda, k(t))$ в виде

$$
S_{t}(\lambda, k(t))=-t\left(m_{0}(\lambda)+m_{1}(\lambda)+i(u, \lambda)\right)-i(\alpha(t), \lambda),
$$

где

$$
m_{0}(\lambda)=1-\beta \sum_{j=1}^{d} \cos \lambda_{j}, \quad \sum_{j=1}^{d}\left|u_{j}\right|<\frac{1}{|\ln \beta|}, \quad \alpha_{j}(t)=o(\sqrt{t}), \quad j=1, \ldots, d .
$$

В силу пространственной однородности рассматриваемой модели можно считать, что вектор $u$ лежит в области, где $u_{j} \geq 0, j=1, \ldots, d$.

Введем далее функцию

$$
S_{0}(\lambda, u)=-m_{0}(\lambda)-i(u, \lambda)
$$

и построим перевальную поверхность для интеграла

$$
\int_{T^{d}} \tilde{h}_{1}^{+}(\lambda) e^{t S_{0}(\lambda, u)} d \lambda
$$

Напомним, что метод перевала состоит из двух частей. Сначала необходимо найти критические точки функции $S_{0}(\lambda, u)$, а затем доказать, что существует перевальная поверхность $\Gamma$, проходяшая хотя бы через одну из этих точек, причем исходная область интегрирования (в данном случае $\lambda \in T^{d}$ ) может быть непрерывно деформирована в поверхность $\Gamma$ так, что соответствуюшие интегралы будут равны

$$
\int_{T^{d}} \tilde{h}_{1}^{+}(\lambda) e^{t S_{0}(\lambda, u)} d \lambda=\int_{\Gamma} \tilde{h}_{1}^{+}(\lambda) e^{t S_{0}(\lambda, u)} d \lambda
$$

ПРЕДЛОЖЕнИЕ 2. Перевальная поверхность Г для интеграла (33) лежит внутри области $W_{\beta}$.

ДокАЗАТЕльСтво. Рассмотрим одномерный случай $d=1$. Из представления (32) для функции $S_{0}(\lambda, u)$ легко видеть, что при всех достаточно малых $\beta, 0<\beta<\beta_{0}(d)$, критические точки $\lambda_{0}^{(k)}(u, \beta), k=1,2$, функции $S_{0}(\lambda, u)$ лежат внутри области $W_{\beta}$. Причем в случае, если $\beta^{1-\varepsilon}<\sum_{j=1}^{d}\left|u_{j}\right|<\frac{1}{|\ln \beta|}$, где $0<\varepsilon<1$, критические точки $\lambda_{0}^{(k)}(u, \beta)$ допускают следуюшее представление:

$$
\lambda_{0}^{(1)}(u, \beta)=-i\left(\ln \frac{2 u}{\beta}+\delta^{(1)}(u, \beta)\right), \quad \lambda_{0}^{(2)}(u, \beta)=\pi+i\left(\ln \frac{2 u}{\beta}+\delta^{(2)}(u, \beta)\right),
$$


где $\left|\delta^{(k)}(u, \beta)\right|<C(\beta / u)^{2}, k=1,2$.

Далее, для построения перевального контура Г воспользуемся тем его свойством, что в окрестности точки перевала он идет по направлению линии уровня функции $\operatorname{Im} S_{0}(\lambda, u)$, а в самой критической точке, лежащей на этом контуре, должен достигаться максимум функции $\operatorname{Re} S_{0}(\lambda, u)$ на контуре. Другими словами, искомый контур должен являться линией наибыстрейшего спуска функции $\operatorname{Re} S_{0}(\lambda, u)$. Анализ функций $\operatorname{Re} S_{0}$ и $\operatorname{Im} S_{0}$ в окрестностях точек $\lambda_{0}^{(k)}(u, \beta), k=1,2$, показывает, что искомый контур Г должен проходить через точку $\lambda_{0}^{(1)}$ и иметь в этой точке горизонтальную касательную. Например, в качестве перевального контура можно взять контур $\Gamma$, проходящий через точку $\lambda_{0}(u, \beta)=\lambda_{0}^{(1)}$ параллельно исходному контуру $T$. При этом в силу аналитичности подьнтегральной функции справедливо равенство (34). Случай больших размерностей $d>1$ рассматривается аналогично. Предложение доказано.

Из предложения и теоремы Руше следует, что при всех достаточно больших $t$ у функции $S_{t}(\lambda, k(t))$ сушествует точка перевала $\lambda_{\mathrm{cr}}(t) \in W_{\beta}$ :

$$
\lambda_{\mathrm{cr}}(t)=\lambda_{0}(u, \beta)+\zeta(u, \beta)+\omega(u, t),
$$

где $\lambda_{0}(u, \beta)$ - точка перевала для интеграла $(33)$, а функции $\zeta(u, \beta)$ и $\omega(u, t)$ удовлетворяют оценкам

$$
\left|\zeta_{j}(u, \beta)\right|<C \beta^{2}, \quad \omega(u, t)=o\left(\frac{1}{\sqrt{t}}\right), \quad j=1, \ldots, d .
$$

При этом перевальная поверхность $\Gamma_{S}$ для интеграла (31) лежит в малой окрестности $\mathcal{U}(\Gamma)$ поверхности $\Gamma$, построенной выше в предложении 2.

Обозначим через $\mu_{\mathrm{cr}} \in W_{\beta}$ вектор

$$
\mu_{\mathrm{cr}}=\lambda_{0}(u, \beta)+\zeta(u, \beta)
$$

Воспользовавшись далее равенством (34), мы находим асимптотическое выражение для интеграла (31) при $t \rightarrow \infty$ по обшей формуле

$$
\int_{\Gamma_{S}} \tilde{h}_{1}^{+}(\lambda) \exp \left\{S_{t}(\lambda, k(t))\right\} d \lambda=\frac{K_{d}}{t^{d / 2}} \exp \left\{S_{t}\left(\mu_{c r}, k(t)\right)\right\}(1+o(1)),
$$

где

$$
K_{d}=\frac{(2 \pi)^{d / 2} \tilde{h}_{1}^{+}\left(\mu_{\mathrm{cr}}\right)}{\left(\left|\operatorname{det}\left\{\left.m^{\prime \prime}(\lambda)\right|_{\mu_{\mathrm{cr}}}\right\}\right|\right)^{1 / 2}} .
$$

Далее, пользуясь аналитичностью функций $\tilde{h}_{1}^{+}(\lambda)$ и $m(\lambda)$ внутри области $W_{\beta}$, а также тем, что

$$
\operatorname{det}\left\{\left.m^{\prime \prime}(\lambda)\right|_{\mu_{\text {cr }}}\right\} \neq 0,
$$

приходим к асимптотической формуле (31). Лемма 3 доказана. 
ЗАмЕчАниЕ 3 . В силу условий $(3)-(5)$ на вектор $k(t)$ мы можем перейти к переменным $t^{\prime}=t /|\ln \beta|, v=u|\ln \beta|$. Тогда экспоненциальный член в формуле (35) будет иметь порядок не больше чем

$$
O\left(\left(C(v) \beta^{(1+\beta)}\right)^{t^{\prime}}(\beta|\ln \beta|)^{t^{\prime} \sum_{j=1}^{d}\left|v_{j}\right|}\right)
$$

где $C(v)$ - константа, зависяшая только от вектора $v \in R^{d}$, а $\sum_{j=1}^{d}\left|v_{j}\right|<1$. Тот же асимптотический вид будет иметь и второе слагаемое в разложении (30). С другой стороны, последнее слагаемое этого разложения имеет порядок $O\left(\beta^{\left(2-C_{2} \beta\right) t^{\prime}}\right)$. Следовательно, при всех достаточно малых $\beta, 0<\beta<\beta_{0}(d)$, выражение (35) определяет главньй член асимптотического выражения для $I_{f, g}(t, k(t))$.

Из этого замечания, а также из (28), (30) и (31) следует формула (6). Теорема 1 доказана.

Автор выражает глубокую благодарность профессору Р. А. Минлосу за полезные обсуждения. Работа выполнена при поддержке Российского фонда фундаментальных исследований, грант № 96-01-00064.

\section{Список литературы}

[1] R. A. Minlos. Markov Processes and Related Fields. 1996. V. 2. № 2. P. 263.

[2] Yu. G. Kondratiev, R. A. Minlos. J. Stat. Phys. (in press).

[3] В. А. Мальиев, Р. А. Минлос. Линейные операторы в бесконечночастичных системах. М.: Наука, 1994.

[4] R. A. Minlos, E. A. Zhizhina. J. Stat. Phys. 1996. V. 84. № 1/2. P. 85.

[5] S. Albeverio, Yu. G. Kondratiev, M. Rockner. J. Funct. Anal. 1995. V. 133. P. 10.

[6] R. Holley, D. Stroock. J. Funct. Anal. 1981. V. 42. P. 29.

[7] М. В. Федорюк. Метод перевала. М.: Наука, 1977.

Поступила в редакцию 8.VIII.1996 г.

\section{E. A. Zhizhina \\ ASYMPTOTIC FORMULA FOR CORRELATION \\ DECAY IN THE STOCHASTIC MODEL OF PLANAR ROTATORS AT HIGH TEMPERATURES}

We found the asymptotic formula for correlations decay $\left\langle f_{A}(x(0)), g_{A+k(t)}(x(t))\right\rangle$, when $t \rightarrow \infty, k(t) \rightarrow \infty, k(t) \in Z^{d}$, in the stochastic model of planar rotators on a lattice $x(t)=\left\{x_{k}(t)\right.$, $\left.k \in Z^{d}\right\}, t \geq 0, x_{k}(t) \in T^{1}$ at high temperatures. The basic methods we use are the spectral analysis of the Markov semigroup generator and the saddle-point method. 